\title{
Orbital cellulitis complicating isolated unilateral sphenoidal sinusitis: importance of the CT scan
}

\author{
C ROBERTS,' A E NYLANDER, ${ }^{2}$ AND S JAYARAMACHANDRAN'
}

From the Departments of 'Otolaryngology and ${ }^{2}$ Ophthalmology, North Staffordshire Royal Infirmary, Stoke on Trent ST4 7LN

SUMMARY A case of orbital cellulitis complicating the rare isolated unilateral sphenoidal sinusitis is reported, stressing the importance of a CT scan where plain $x$-rays fail to show any abnormality.

Orbital cellulitis may result from different septic foci, sinusitis being a common source. Treatment must be directed towards the source of infection. Orbital cellulitis as a complication of isolated unilateral sphenoidal sinusitis is very rare. Underlying sinusitis in cases of orbital cellulitis is usually diagnosed with plain $x$-rays. However, plain $x$-rays may prove inadequate in cases of sphenoidal sinusitis, for they may fail to show the abnormality, so that correct treatment may be delayed. This risk can be avoided by promptly performing a CT scan.

\section{Case report}

A 71-year-old Caucasian woman presented with a sore, swollen, tender left eye of three days' duration. She was receiving treatment to both eyes for chronic simple glaucoma (timolol maleate and dipivefrin hydrochloride). For the previous six years she had received oral prednisolone $10 \mathrm{mg}$ daily for rheumatoid arthritis. For three months she had been on rifampicin, isoniazid, and pyridoxine for pulmonary tuberculosis.

At presentation she was apyrexial. There was massive left orbital swelling, with very injected conjunctivae and chemosis but no lymphadenopathy. The cornea was clear, and the intraocular pressure was raised at $34 \mathrm{~mm} \mathrm{Hg}$. The pupil was enlarged and reacted sluggishly to light. The retina showed no choroidal folds or other abnormality. There was no proptosis. Her visual acuity was $6 / 6$ right eye and hand movements left eye (recorded 6/9 one month previously).

Plain $x$-rays of the paranasal sinuses (Fig. 1) were

Correspondence to Mr C Roberts. reported by an experienced radiologist as normal. Treatment was begun with intravenous ampicillin and flucloxacillin (250 $\mathrm{mg}$ of each six hourly). Despite 24 hours of treatment there was increasing orbital swelling. A CT scan of the orbits and sinuses

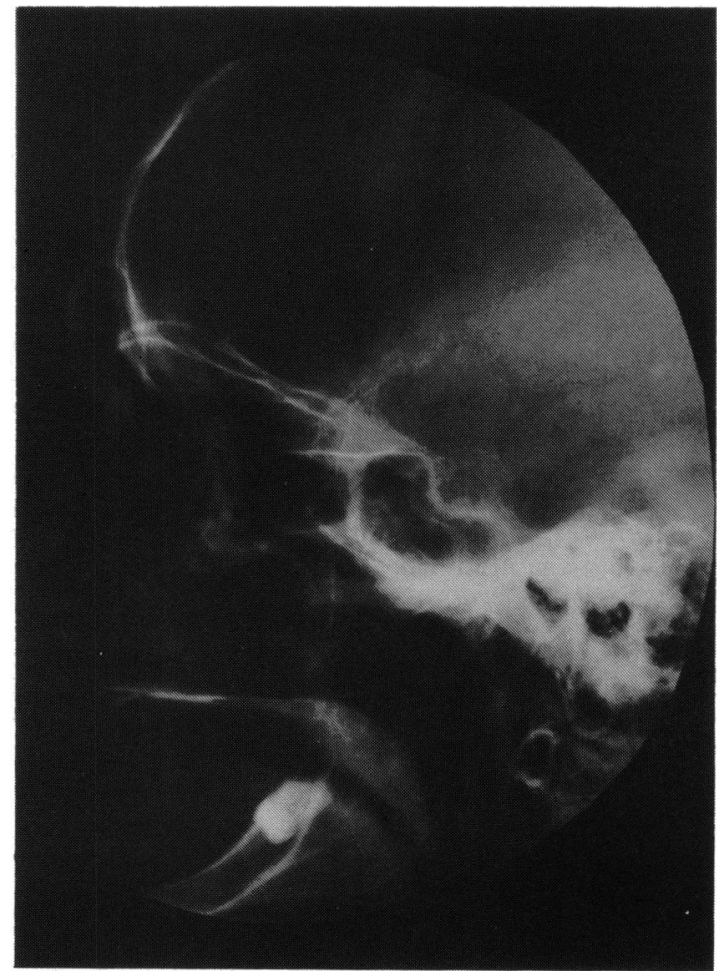

Fig. 1 Lateral $\mathrm{x}$-ray. Sphenoidal sinuses of normal appearance. 


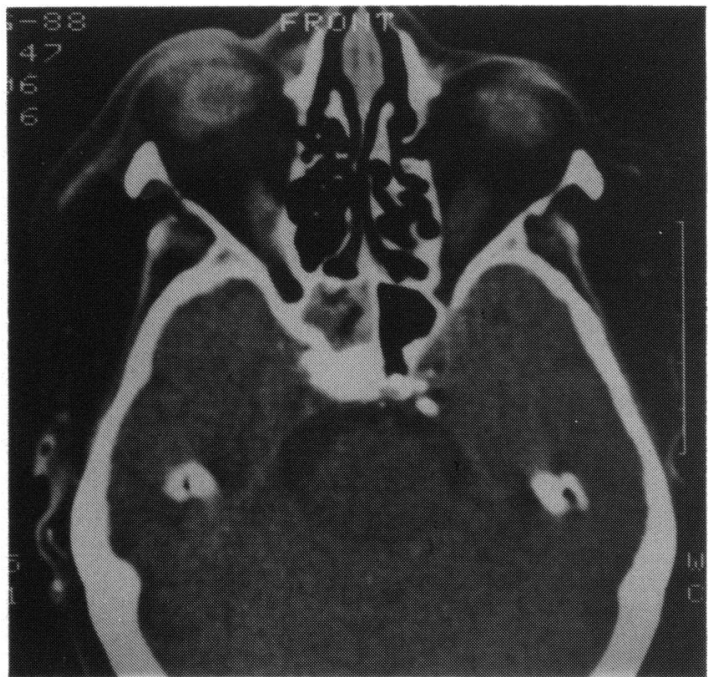

Fig. 2 Computerised axial tomography showing opacification of the left sphenoidal sinus.

was performed. This showed opacification of the left sphenoid sinus (Fig. 2). Her antibiotic dosage was therefore doubled and the opinion of an otolaryngologist sought.

The left sphenoethmoid complex was approached through a Howarth incision. The anterior, middle, and posterior groups of ethmoidal cells were found to be healthy. The sphenoid sinus, however, was found to be lined by thickened mucosa and filled with inspissated mucus. All diseased mucosa was cleared, and the lateral wall of the middle meatus was widely excised to allow adequate drainage of the surgically created sphenoethmoidal cavity.

No organism could be isolated despite prolonged culture of samples of both mucosa and mucus. Histological examination of the mucosa revealed chronic inflammation. No granuloma or caseation was found.

After the operation the cellulitis resolved in 48 hours and vision improved to $6 / 18$ (left eye) at two weeks, settling to $6 / 12$ at four months.

\section{Discussion}

Since the days of Hippocrates sinusitis has been recognised as a common cause of orbital cellulitis. ${ }^{1}$
The source of infection is usually the ethmoid, followed in frequency by the maxillary and frontal sinuses.' Though well recognised, isolated sphenoidal sinusitis is rarely the cause, and rarer still are reports of isolated unilateral sphenoidal sinusitis causing orbal cellulitis. ${ }^{\text {: }}$

Dale and Mackenzie ${ }^{2}$ reported on a series of five patients with complications of sphenoidal sinusitis in whom the diagnosis was easily made with plain $x$ rays. These should include the submento vertical and lateral views to demonstrate the sphenoid sinuses, so that the rare, but important, diagnosis of sphenoidal sinusitis is not missed. ${ }^{-4}$ However, there is a $15 \%$ incidence of failure to detect sphenoidal sinusitis with plain $x$-rays. ${ }^{5}$ Plain $x$-rays may therefore allow the correct diagnosis to be made but cannot be relied upon to exclude underlying sphenoidal sinusitis, as found in our patient.

In orbital cellulitis CT scan is usually considered for patients who fail to respond to medical treatment. "Early diagnosis and treatment are essential to minimise the risk of severe visual loss. ${ }^{7}$ Surgical intervention is required early in cases with underlying sinusitis to minimise this risk. ${ }^{8}$ Our dependence on plain $x$-rays delayed the diagnosis of the underlying pathology and hence necessary surgical intervention. Fortunately there was no serious visual loss. When no cause can be be found on plain $x$-rays of the paranasal sinuses, CT scan can be useful in establishing the diagnosis and avoiding delay in giving the correct treatment.

\section{References}

1 Shahin J, Gullane PJ, Dayal VS. Orbital complications of acute sinusitis. J Otolaryngol 1987; 16: 23-7.

2 Dale BA, Mackenzie IJ. The complications of sphenoid sinusitis. J Laryngol Otol 1983; 97: 661-70.

3 Levine H. The sphenoid sinus, the neglected nasal sinus. Arch Otolaryngol 1978; 104: 585-7.

4 Gray WC, Blanchard CL. Sinusitis and its complications. Am Fum Physician 1987; 35: 232-43.

5 Wyllie JW, Kern EB, Djalilian M. Isolated sphenoid sinus lesions. Laryngoscope 1973; 83: 1252-65.

6 Lawless M, Martin F. Orbital cellulitis and preseptal cellulitis in childhood. Aust NZ J Ophthalmol 1986; 14: $211-9$.

7 Reidy JJ, Giltner J, Apple DJ, Anderson RL. Paranasal sinusitis, orbital abcess, and inflammatory tumors or the orbit. Ophthalmic Surg 1987; 18: 363-6.

8 Mills RP. Orbital infection and sinusitis. $J R$ Soc Med 1986; 79: 68-9.

Accepted for publication 3 April 1989. 